\title{
Responsabilidad social corporativa: factores influyentes en la divulgación de información en organizaciones de salud no lucrativas
}

Cómo citar este artículo / To reference this article / Comment citer cet article / Para citar este artigo:

Zanfrillo, A. I.; Artola, M. A. (2021). Responsabilidad social corporativa: factores influyentes en la divulgación de información en organizaciones de salud no lucrativas. Revista Escuela de Administración de Negocios, (90), 139-152. DOI: https://doi. org/10.21158/01208160.n90.2021.2930

\section{Resumen}

Conscientes de la importancia de mejorar las condiciones de vida de la comunidad a través del desarrollo de hábitos saludables, las organizaciones no lucrativas de salud argentinas se enfrentan al desafío de ser sostenibles en un contexto inflacionario elevado y de ingresos limitados a los aportes salariales de sus afiliados, a fin de optimizar las demandas prestacionales. En el marco de la responsabilidad social corporativa (RSC), las acciones que favorecen el cuidado de la salud se sitúan en un lugar destacado para desarrollar la concientización de los afiliados sobre los comportamientos responsables individuales y colectivos. Ante este panorama, el propósito del trabajo consiste en analizar los factores influyentes en la divulgación de la dimensión social de la RSC en las entidades no lucrativas prestadoras o financiadoras de servicios de salud de la ciudad de Mar del Plata — República Argentina - como instrumento para desarrollar estrategias dirigidas a la promoción de la salud en la comunidad. La investigación cuantitativa fue efectuada sobre la publicación de contenidos en los medios digitales a través de un modelo de regresión categórica, lo que permitió distinguir la relevancia de los atributos organizativos frente a los tecnológicos, vinculando el fomento de prácticas saludables con la naturaleza de estas entidades.

Palabras clave: responsabilidad social corporativa; promoción de la salud; organizaciones de salud no lucrativas; compromiso social; presencia en internet; canal de información digital; comunicación digital.

1 Licenciada en Sistemas - Universidad CAECE. Magister en Gestión Universitaria - Universitat de les Illes Balears. Doctora en tecnología educativa: aprendizaje virtual y gestión del conocimiento - Universitat Rovira i Virgili. Directora del Grupo de Investigación Gestión de Información, Sistemas y Tecnologías y del Programa Tecnologías y Responsabilidad Social de la Facultad de Ciencias Económicas y Sociales de la Universidad Nacional de Mar del Plata, Argentina. ORCID: https://orcid.org/0000-00033638-2672

2 Contadora Pública y Magister en Administración de Negocios - Universidad Nacional de Mar del Plata. Codirectora del Grupo de Investigación Gestión de Información, Sistemas y Tecnologías y del Programa Tecnologías y Responsabilidad Social de la Facultad de Ciencias Económicas y Sociales de la Universidad Nacional de Mar del Plata, Argentina. ORCID: https://orcid.org/0000-00030662-7042 


\title{
Corporate social responsibility: factors influencing information disclosure in non-profit health organizations
}

\begin{abstract}
Being aware of the importance of improving the living conditions of the community throughout the development of healthy habits, Argentinian non-profit health organizations face the challenge of being sustainable in a context of high inflation and an income that is limited to the salary contributions of their members, in order to optimize the demands of their services. Within the framework of Corporate Social Responsibility (CSR), the actions that favor health care are in a prominent place as they develop members' awareness of individual and collective responsible behaviors. Faced with this background, the purpose of this study is to analyze the factors influencing the dissemination of the social dimension of CSR in non-profit organizations providing or financing health services in the city of Mar del Plata, Argentina, as an instrument for developing strategies that are aimed at promoting health in the community. A quantitative research was carried out on the publication of content in digital media through a categorical regression model, which made it possible to distinguish the relevance of organizational attributes versus technological attributes, linking the promotion of healthy practices with the nature of these entities.
\end{abstract}

Keywords: corporate social responsibility; health promotion; non-profit health organizations; social commitment; internet presence; digital information channel; digital communication.

\section{Responsabilidade social empresarial: fatores que influenciam na divulgação de informações em organizações de saúde sem fins lucrativos}

\section{Resumo}

Conscientes da importância de melhorar as condições de vida da comunidade por meio do desenvolvimento de hábitos saudáveis, as entidades argentinas de saúde sem fins lucrativos enfrentam o desafio de serem sustentáveis em um contexto inflacionário alto e de renda limitada às contribuições salariais de seus funcionários, para otimizar as demandas de serviços. No âmbito da Responsabilidade Social Empresarial (RSE), as ações que privilegiam a assistência à saúde ocupam um lugar de destaque para desenvolver a consciência dos funcionários sobre comportamentos responsáveis individuais e coletivos. Neste contexto, o objetivo do trabalho é analisar os fatores que influenciam a difusão da dimensão social da RSE em entidades sem fins lucrativos que prestam ou financiam serviços de saúde na cidade de Mar del Plata República Argentina - como instrumento para desenvolver estratégias voltadas para a divulgação da saúde na comunidade. A pesquisa quantitativa foi realizada sobre a publicação de conteúdos em mídias digitais por meio de um modelo de regressão categórica, que permitiu distinguir a relevância dos atributos organizacionais em relação aos tecnológicos, vinculando a promoção de práticas saudáveis à natureza dessas entidades.

Palavras-chave: responsabilidade social empresarial; divulgação da saúde; organizações de saúde sem fins lucrativos; compromisso social, presença na internet; canal de informação digital; comunicação digital. 


\section{$\longrightarrow \quad$ Responsabilité sociale des entreprises : facteurs d'influence dans la divulgation d'informations des organisations de santé à but non lucratif}

\section{Résumé}

Conscientes de l'importance d'améliorer les conditions de vie des communautés par le développement d'habitudes de vie saines, les organisations de santé argentines à but non lucratif doivent pérenniser leurs activités et optimiser les performances de leurs prestations dans un contexte de forte inflation et de faibles cotisations salariales des affiliés. Dans le cadre de la responsabilité sociétale des entreprises (RSE), les actions en faveur des soins de santé ont une place prépondérante pour sensibiliser les adhérents à des comportements individuels et collectifs responsables. L'objectif de cet article sera d'analyser les facteurs influençant la diffusion de la dimension sociale de la RSE dans les entités à but non lucratif offrant ou finançant des services de santé dans la ville de Mar del Plata — République d'Argentine — comme instrument de développement stratégique d'offre de services de soins à la communauté. La recherche quantitative est le fruit de publication de contenu dans des médias numériques, à travers un modèle de régression catégorielle, distinguant la pertinence des attributs organisationnels et technologiques, reliant la promotion de pratiques saines à la nature des entités.

Mots-clés: responsabilité sociale des entreprises; santé; organismes de santé à but non lucratif; compromis social; présence sur internet; canal d'information numérique; communication numérique. 


\section{Introducción}

$\mathrm{L}$ os escándalos empresariales ocurridos en las últimas décadas del siglo pasado han puesto de manifiesto el interés creciente de la sociedad por el comportamiento ético de las organizaciones (Izquierdo y Grañana, 2005). La comunicación de una conducta responsable, de valores y pautas de actuación se constituyen en un pilar fundamental de los nuevos modelos de gestión que pretenden un equilibrio entre las dimensiones económica, social y ambiental para mejorar tanto su propio desempeño como el del entorno en el que llevan a cabo su actividad (Mateos-Espejel, EstradaRodríguez y Hernández-Ruiz, 2020; MuñozMartín, 2013).

La importancia que le asignan los consumidores a la conducta responsable de las organizaciones y la pronta penalización que realizan sobre aquellas que detentan un comportamiento contrario a estos lineamientos señalan la divulgación de contenidos sobre RSC como un factor capaz de consolidar la reputación de la organización y constituirse en una fuente de ventaja competitiva (Ajina et al., 2019; Alvarado-Herrera y Schlesinger-Díaz, 2008; Moreno y Capriotti, 2006).

Dada la importancia de internet como medio para comunicar sus prácticas, las organizaciones publican diferentes tipos de temáticas relacionadas con la RSC y los recursos que provean interacción con sus stakeholders. En este sentido, la web 2.0 se ha reconocido con la denominación de accountability web por constituir un canal informativo dirigido a ofrecer transparencia en las acciones y la rendición de cuentas, para obtener así beneficios y externalidades adicionales (Baue y Murningham, 2011).

En la República Argentina las entidades no lucrativas proveen diferentes servicios, en particular, cobertura sanitaria a los sectores más desprotegidos de la población en un sistema de salud escasamente integrado entre los sectores que lo componen y fragmentado en su interior (Belló y Becerril-Montekio, 2011; Sigliano, 2013). Estas limitaciones, sumadas a las crisis económicas, generaron mayores dificultades para el acceso a una cobertura de salud, estableciendo una $\ll[\ldots]$ brecha entre los servicios ofrecidos y las necesidades de los usuarios $\gg$ (Sigliano, 2013, p. 1). Pues, teniendo en cuenta el carácter estratégico de la comunicación de RSC con los grupos de interés y el interés de las organizaciones de salud en la promoción de la salud (Koinig, Diehl y Mueller, 2017; Organización Mundial de la Salud, 1986; Rodríguez-Cala et al., 2017) asociado con el rol de las entidades no lucrativas de $\ll$ motor de búsqueda» para el desarrollo de soluciones a los problemas actuales de la sociedad (Anheier, 2013), se considera significativo el abordaje de la difusión de las acciones de estas entidades y la interacción con sus grupos de interés a través de los medios digitales.

Este trabajo aborda el estudio de los factores que inciden en la publicación sobre la divulgación de RSC, para las organizaciones sin fines de lucro dedicadas a la prestación o financiación de servicios de salud en Mar del Plata República Argentina-. Se plantean los siguientes interrogantes que guían la investigación: ¿cómo es la divulgación de contenidos sobre compromiso social en las entidades no lucrativas vinculadas con la salud?, y, zqué elementos influyen en la publicación de contenidos sobre su preocupación social? La estructura del documento comprende: a) la temática de RSC y su comunicación, b) la metodología a adoptar, c) los resultados del análisis descriptivo y multivariante, y d) los principales temas para discusión sobre las hipótesis planteadas y la importancia de la comunicación de la RSE en este tipo de entidades que integran el sistema de salud argentino. 


\section{Aspectos conceptuales}

a RSC se define como las acciones que llevan
adelante las empresas en respuesta a los requerimientos de sus grupos de interés (Freeman, 1984), a fin de incrementar el valor de marca y la reputación (Benavides-Delgado, FernándezFernández y de Vivar-Fontelo, 2012). El origen del término responsabilidad social se debe a Bowen (1953), quien extiende las responsabilidades de los empresarios más allá de los beneficios económicos de las empresas, hacia la adquisición de compromisos orientados a impulsar políticas para desarrollar el bienestar de la comunidad.

Según el tamaño de la organización, en la literatura académica se aborda la responsabilidad social empresaria o la responsabilidad social corporativa, en atención a grandes empresas - en el último caso- y a aquellas que sin ser lucrativas se asemejan, en algunos aspectos, como, por ejemplo, las cajas de ahorro y las organizaciones no gubernamentales, entre otras (Benavides-Delgado et al., 2012).

Si bien las organizaciones no lucrativas se enmarcan en la RSC, se observa la necesidad de plantear un nuevo término que englobe las acciones responsables que desarrollan este tipo de entidades (Waters y Ott, 2014). La ambigüedad y complejidad atribuida a la responsabilidad social corporativa es reconocida por diferentes autores, lo que genera una falta de consenso para su definición (Džupina y Džupinová, 2019; Mateos-Espejel et al., 2020; Muñoz-Martín, 2013; Sánchez-Hervás y Subiela-Hernández, 2016).

En la RSC se distinguen diferentes responsabilidades - morales, sociales y legales - que afectan a las organizaciones y obligan así a las empresas a ser transparentes en sus prácticas de gestión, a actuar en consonancia con las expectativas que la sociedad plantea a su accionar, en un marco de valores acorde con la época y el contexto (Muñoz-Martín, 2013). La evolución del concepto de RSC se aleja de una conducta filantrópica a su integración en la estra- tegia organizacional con impacto sobre los grupos de interés, en los aspectos económicos, sociales y medioambientales reconocidos en las personas, las ganancias y el ambiente a través del triple impacto (Carroll, 1979; Muñoz-Martín, 2013).

Para las organizaciones no lucrativas, como, por ejemplo, las cooperativas, la responsabilidad social no tiene carácter voluntario, sino que es un principio constitutivo, una obligación ética hacia sus grupos de interés y con el público en general, mediante un accionar responsable con sus asociados y la sociedad en general (VargasSánchez y Vaca-Acosta, 2005). Mientras que para el sector industrial la RSC tiene como propósito la reducción de riesgos derivados de problemas de seguridad, la disminución del impacto ambiental negativo, la captación de talentos y la consolidación de una reputación positiva (Wirth, Kulczycka, Hausner y Koński, 2016). Ahora, para las entidades no lucrativas, basadas en el bienestar social, esta conducta les es inherente, aunque no así su divulgación.

Las empresas de economía solidaria baleares no ofrecen información de forma voluntaria con, aproximadamente, la publicación de la mitad de los contenidos descriptos en los estándares del Global Reporting Initiative (Horrach y Socias-Salvà, 2011). Para las ONG españolas se ofrece una visión aún más desalentadora: registran un desempeño inferior a las empresas IBEX-35 con una presencia expositiva y escasa interacción (Tapia-Frade y Caerols-Mateo, 2015). Esta situación también se revela en algunos casos de análisis, como en una cooperativa de crédito española que presenta en internet un limitado número de contenidos sobre RSC, falta de estándares y de instancias de diálogo con los públicos de interés (Seguí-Mas, SarasaPérez y Baviera-Puig, 2012). 
La RSC tiene en la comunicación un pilar fundamental para dar cuenta de su quehacer a través de información confiable que brinde equilibrio a las interacciones que desarrollan con sus públicos objetivos, pues además de efectuar las acciones es necesario que las comuniquen estratégicamente a fin de proyectar los procesos internos hacia el exterior y así reflejar la imagen de la empresa, considerando vital la integración del público interno, en primer lugar, con la finalidad de cohesionar comportamientos y estructurar conductas (Castaño-González, 2011).

En la comunicación de la RSC se plantea la desconfianza de los grupos de interés, pues si bien quieren conocer las buenas acciones realizadas, también ponen en duda las motivaciones para su desarrollo, planteando como desafío superar el escepticismo y generar atribuciones genuinas o intrínsecas en las que se considera que la actuación se basa en una preocupación legítima (Du, Bhattacharya y Sen, 2010).

Si bien internet se constituye en el medio principal para la divulgación de información sobre RSC a los grupos de interés y la web corporativa es el vehículo que permite difundir buenas prácticas e informes de sostenibilidad, los contenidos que se presentan resultan limitados, la comunicación es unidireccional y escasamente se presentan criterios externos a fin de garantizar el comportamiento corporativo que se difunde (Moreno y Capriotti, 2009; Rodríguez-Cala et al., 2017; Sánchez-Hervás y Subiela-Hernández, 2016). En algunos países, para las empresas que cotizan en el mercado de valores, resulta de obligatoriedad publicar en la web la disposición sobre estados financieros, actas de reuniones y datos de contacto, entre otros temas de importancia bajo un formato particular (Álvarez, Sánchez y Domínguez, 2009).

Con respecto a la comunicación de RSC, las entidades no lucrativas ofrecen un panorama con escasa visibilidad de sus acciones a través del medio digital y limitadas posibilidades de retroalimentación con los públicos de interés, de modo que dan preferencia a otros canales para divulgar su comportamiento en materia de responsabilidad social (Waters y Ott, 2014). Otras investigaciones en organizaciones de carácter no lucrativo dan cuenta de la ausencia en materia de contenidos sobre RSC, en particular, la limitación de contenidos expuestos, la falta de adhesión a estándares internacionales y la escasa interacción con los grupos de interés (Gazzola, Ratti y Amelio, 2017; Seguí-Mas et al., 2015).

En el ámbito hospitalario catalán, si bien consideran que la web es un instrumento comunicativo imprescindible, encuentran cierta variabilidad en la información publicada, accediendo así a una fase inicial de información sobre RSC y sin interacción con los grupos de interés (Rodríguez-Cala et al., 2017). Las investigaciones analizadas muestran una fase inicial en el uso de la web corporativa para la divulgación de sus acciones en materia de RSC, con una presencia incipiente y escasas herramientas de interacción. Esto dificulta el conocimiento de su accionar por parte de los grupos de interés más jóvenes o de uso intensivo de las tecnologías.

El análisis de la literatura científica plantea limitaciones en el acceso a investigaciones sobre la comunicación de RSC para entidades no lucrativas de salud, mas no referida a centros y hospitales, sino a las organizaciones que gestionan estas prestaciones, dado que existen tanto para centros de salud como para ONG o instituciones religiosas de forma individual, pero no para las entidades sin fines de lucro que prestan o financian estos servicios, propias del sistema de salud argentino. 


\section{Metodología}

El propósito del estudio consiste en determinar los factores que inciden sobre la divulgación de la dimensión social de la RSC a través de las tecnologías de internet para las organizaciones de salud radicadas en Mar del Plata. Se abordó un diseño cuantitativo, de tipo exploratoriocorrelacional a partir del relevamiento de 30 entidades no lucrativas de salud en el 2019, con presencia en internet y definidas en su identidad legal como asociaciones, mutuales o fundaciones, las cuales se distribuyeron mayoritariamente entre las dos primeras categorías (véase la Tabla 1).

La elección de este colectivo se fundó en la diversidad y el volumen de entidades prestadoras o financiadoras de servicios de salud enmarcadas en el tercer sector que integran el ámbito privado del sistema de salud argentino y atienden a un vasto público suscripto por elección voluntaria, financiando y proporcionando prestaciones sanitarias de diferente cobertura y complejidad a sus afiliados (Belló y Berrecil-Montekio, 2011). Otro factor de interés se basó en el rol de las entidades sin fines de lucro frente a las problemáticas sociales, consideradas como «motor de búsqueda» para ofrecer soluciones en forma innovadora en contextos económicos cada vez más complejos e inciertos (Anheier, 2013).

Tabla 1. Composición de entidades del tercer sector vinculadas con los servicios de salud, Mar del Plata, 2019

\begin{tabular}{|c|c|c|}
\hline Tipo de entidad & Cantidad & $\mathbf{\%}$ \\
\hline Asociación & 13 & 43,3 \\
\hline Fundación & 2 & 6,7 \\
\hline Mutual & 15 & 50,0 \\
\hline Total & $\mathbf{3 0}$ & $\mathbf{1 0 0 , 0}$ \\
\hline
\end{tabular}

Fuente. Elaboración propia.

Las entidades del estudio se identificaron a través de la búsqueda en la Federación de Fundaciones Argentina, la Confederación Argentina de Mutualidades, la Superintendencia de Servicios de
Salud y el Instituto Nacional de Asociativismo y Economía Social, con un total de 58 organizaciones. Se corroboró que cumplieran los siguientes criterios: a) entidad no lucrativa, b) prestación de servicios de salud como actividad económica principal o secundaria constatada a través de la Administración Federal de Ingresos (AFIP), c) domicilio principal o de una de sus sedes en Mar del Plata, y d) presencia digital a través de un sitio web localizado a través de motores de búsqueda, de modo que abarca a partir de la incorporación de este último criterio un total de 30 organizaciones.

El relevamiento se llevó a cabo en el mes de mayo del 2019 por dos investigadores para el reconocimiento y la validación cruzada de los contenidos identificados en el ámbito digital a partir del consenso de criterios para la ubicación y la codificación, así como la búsqueda de información general sobre las entidades en fuentes de información secundaria.

Sobre cada website correspondiente a las entidades en estudio se aplicó la técnica de análisis de contenido como uno de los métodos más empleados en la investigación en comunicación (Berger, 2020). El análisis de los website se efectuó de forma objetiva y sistemática al disponer de pautas para la extracción de los contenidos requeridos en las fuentes seleccionadas (Duverger, 1981). Estas pautas se asocian a las variables de investigación, es decir, en los contenidos publicados se realizó la búsqueda sobre la definición del objeto social, los servicios o naturaleza de las prestaciones, el ámbito de actuación, el uso de dominios específicos para la difusión de su presencia digital y los contenidos sobre compromiso social y difusión de los perfiles en redes sociales (véase la Tabla 2). Esta herramienta de análisis permite clasificar el contenido de las distintas secciones del website a fin de extraer la información relevante o las tendencias predominantes en forma de categorías (Pardinas, 2005). 
Para la definición de los factores que inciden en la publicación de contenidos se seleccionaron aquellos relacionados con la dimensión social y otros bajo las categorías de organizativos y tecnológicos, adaptados para el diseño metodológico de instrumentos de relevamiento y recogida de datos emergentes de la revisión de la literatura científica sobre la temática de comunicación de RSC (Moreno y Capriotti, 2006; Rodríguez-Cala et al., 2017) (véase la Tabla 2).

Se denominaron factores organizativos tales como identidad legal, naturaleza de las prestaciones y alcance, mientras que se identificaron como factores tecnológicos familiaridad con la tecnología, canales de información digital y recursos de la website. En esta última categoría se consideran para su análisis aquellas publicaciones que fueran declaraciones de intención, objetivos o contenidos propios o replicados de otros ámbitos, para la promoción y prevención de la salud, la gestión de la diversidad, la conciliación de la vida familiar y laboral y proyectos solidarios. Las valoraciones contempladas para la dimensión social sobre RSC fueron dos:

- presente, si la entidad publica información sobre los aspectos sociales enunciados anteriormente;

- ausente, si la entidad no exhibe información en beneficio del bienestar de la población, en favor ni sobre los proyectos solidarios y/o de voluntariado en los que participa.

Tabla 2. Variables del modelo propuesto

\begin{tabular}{|c|c|c|c|}
\hline Variable & Descripción & Indicador & Valores \\
\hline Identidad legal & $\begin{array}{l}\text { Característica distintiva de la entidad } \\
\text { respecto del objeto social. }\end{array}$ & Figura jurídica & Asociación-fundación-mutual \\
\hline $\begin{array}{l}\text { Naturaleza de las } \\
\text { prestaciones }\end{array}$ & $\begin{array}{l}\text { Servicios que brinda la entidad a sus } \\
\text { asociados. }\end{array}$ & Tipo de servicio & $\begin{array}{l}\text { Financiamiento-provisión de } \\
\text { servicios de salud-financiamiento } \\
\text { y provisión }\end{array}$ \\
\hline Alcance & Ámbito de actuación de la entidad. & $\begin{array}{l}\text { Presencia física de la } \\
\text { entidad }\end{array}$ & Local-regional/nacional \\
\hline $\begin{array}{l}\text { Familiaridad con la } \\
\text { tecnología }\end{array}$ & $\begin{array}{l}\text { Uso de dominios específicos para la } \\
\text { difusión de la presencia digital, nacional } \\
\text { o internacional, comercial o para } \\
\text { organizaciones sin fines de lucro. }\end{array}$ & Nombre de dominio & Alta-moderada-baja \\
\hline $\begin{array}{l}\text { Canales de } \\
\text { información digital }\end{array}$ & $\begin{array}{l}\text { Contenidos actualizados en el sitio web } \\
\text { en formato específico para los usuarios. }\end{array}$ & $\begin{array}{l}\text { Contenido para el } \\
\text { asociado }\end{array}$ & $\begin{array}{l}\text { Publicación de contenido-sin } \\
\text { publicación }\end{array}$ \\
\hline $\begin{array}{l}\text { Recursos de la web } \\
\text { social }\end{array}$ & $\begin{array}{l}\text { Disponibilidad de recursos para } \\
\text { desarrollar la presencia digital de la } \\
\text { entidad en la web 2.0. }\end{array}$ & Existencia de perfiles & $\begin{array}{l}\text { Mayor disponibilidad- } \\
\text { moderados-mínimos-sin recursos }\end{array}$ \\
\hline Compromiso social & $\begin{array}{l}\text { Publicación de contenidos referidos } \\
\text { a su compromiso social y cuidado } \\
\text { medioambiental. }\end{array}$ & $\begin{array}{l}\text { Presencia de } \\
\text { contenidos sobre } \\
\text { RSC }\end{array}$ & Presencia-ausencia \\
\hline
\end{tabular}

Fuente. Elaboración propia.

El análisis de los estudios sobre divulgación de información sobre RSC posibilitó la definición de dos hipótesis. La primera vincula los factores organizativos con la publicación de contenidos sobre RSC, y la segunda los factores tecnológicos (Moreno y Capriotti, 2006; Rodríguez-Cala et al., 2017): 
- hipótesis 1: los factores organizativos son relevantes de la publicación digital sobre la dimensión social de RSC en entidades no lucrativas y se definen por los aspectos jurídicos, de actividad económica y cobertura geográfica;

- hipótesis 2: los factores tecnológicos son relevantes de la publicación digital sobre la dimensión social de RSC en entidades no lucrativas y se definen por los conocimientos previos sobre la tecnología, la disposición de canales informativos y el uso de recursos digitales.
A fin de contrastar o refutar las hipótesis planteadas se empleó una técnica de regresión categórica que permite escalar las variables nominales $y$ ordinales seleccionadas, cuantificando sus categorías y obteniendo, finalmente, una ecuación de regresión lineal que representa la influencia de cada factor en la predicción del atributo a explicar. En este modelo se relaciona una variable dependiente correspondiente a los contenidos sobre la dimensión social de RSC con variables independientes relacionadas con factores organizativos y tecnológicos.

\section{Resultados}

\subsection{Análisis descriptivo}

El análisis de los contenidos publicados en los sitios web de las 30 entidades de servicios de salud sobre la dimensión social de RSC mostró una escasa comunicación sobre la temática, de modo que constituye una dificultad crítica para la concientización de los públicos de interés (véase la Tabla 3). En un poco más del $73 \%$ de las entidades en estudio no se publicaron contenidos relacionados con la salud de sus afiliados y la comunidad, bien sea a través de acciones de concientización y formación de hábitos saludables de elaboración propia o por replicación de campañas nacionales o regionales, resultando las mutuales con mayor presencia en relación con las asociaciones y las fundaciones.

Este escaso interés por los requerimientos de la comunidad resulta comparable con estudios en otros países en vías de desarrollo (Adhepeau, 2013), así como en investigaciones sobre organizaciones no lucrativas con limitada publicación de contenidos de RSC y escasa interacción con sus públicos de interés (Gazzola et al., 2017; SeguíMas et al., 2012). Se destaca de esta forma la baja importancia que se le asigna a la difusión de este tipo de contenidos, en particular como tema de relevancia para los diferentes stakeholders que pudieran acceder a la información que brindan a través de internet sobre las medidas preventivas a nivel local y regional.

Del universo de unidades que cumplían con el requisito inicial, estar radicadas en Mar del Plata - provincia de Buenos Aires de la República Argentina - se identificaron 30 entidades con presencia digital sobre un total de 58 no lucrativas de salud. Se observó así que la disposición de un sitio web no resulta una prioridad para este colectivo, con un porcentaje apenas mayor al $50 \%$ de organizaciones sin fines de lucro en este ámbito. Esta situación resulta coincidente con la escasa presencia digital de entidades no lucrativas en países desarrollados que registran las dificultades que se suscitan ante la necesidad de atraer voluntarios y donaciones, así como incorporar en su audiencia segmentos más jóvenes que realizan un uso intensivo de la tecnología y desarrollar una comunicación eficaz con quiénes requieren sus servicios (McMahon, Seaman, Lemley, 2015). 
Tabla 3. Compromiso social en entidades no lucrativas prestadoras y/o financiadoras de servicios de salud. Mar del Plata —República Argentina_, 2019

\begin{tabular}{|c|c|c|}
\hline \multirow{2}{*}{ Compromiso social publicado en internet } & \multicolumn{2}{|c|}{ Entidades } \\
\cline { 2 - 3 } & Cantidad & $\%$ \\
\hline Con presencia & 8 & 26,7 \\
\hline Sin presencia & 22 & 73,3 \\
\hline Total & 30 & 100,0 \\
\hline
\end{tabular}

Fuente. Elaboración propia.

En los resultados de una investigación sobre las organizaciones sin fines de lucro del sector sanitario estadounidense se advierte sobre el menor volumen de información que se divulga para los públicos de interés, aduciendo como una causa probable la falta de incentivos propuestos por el marco normativo que las regula, con una escasa promoción de beneficios para la comunicación de contenidos sobre RSC de carácter voluntario (Tremblay-Boire y Prakash, 2015). Estas situaciones ofrecen una analogía con la escasez de presencia digital de las entidades no lucrativas de salud marplatenses y su bajo nivel de publicación de contenidos sobre RSC.

\subsection{Análisis multivariante}

Con el propósito de determinar los factores relacionados con la divulgación de RSC en internet se aplicó un modelo de análisis de regresión categórica, cuya variable explicada representa la intensidad de la comunicación de temáticas sobre el bienestar de la población a través de las campañas de salud e información de interés para la mejora de la calidad de vida. Se estudió, en primer término, la existencia de correlación entre las variables del modelo. El análisis de correlaciones bivariante muestra relaciones bajas o moderadas, directas e inversas, por lo cual el método puede ser utilizado sin vulnerar los supuestos establecidos.

El modelo, con el 73,3\% de entidades sin presencia de contenidos específicos, ofrece una capacidad explicativa del $74 \%$. El valor de F-statistic es de 6,294 con un valor de significación inferior a 0,01 , por lo cual las variables del estudio tienen influencia en la divulgación de contenidos de RSC con un $99 \%$ de confianza en la capacidad explicativa del modelo propuesto.

En el análisis de significatividad de las variables independientes, tres de ellas, identidad legal, naturaleza de las prestaciones y familiaridad con la tecnología son las que se asocian de forma positiva con respecto a la variable explicada (véase la Tabla 4). Dos de las variables significativas en la explicación de la dependiente corresponden a factores organizativos, identidad legal y naturaleza de las prestaciones, y la restante se inscribe en los factores tecnológicos: familiaridad con la tecnología. Las variables alcance, canal informativo digital y recursos de la web social no resultaron explicativas para el modelo propuesto.

Tabla 4. Modelo de regresión categórica

\begin{tabular}{|c|c|c|c|c|c|}
\hline \multirow{2}{*}{ Modelo } & \multicolumn{3}{|c|}{ Coeficientes de la regresión categórica } & \multirow{2}{*}{$\begin{array}{c}\text { Correlación de } \\
\text { orden cero }\end{array}$} & \multirow{2}{*}{ Importancia } \\
\hline & Beta & $\mathbf{F}$ & Sig. & & \\
\hline Identidad legal & 0,708 & 6,826 & $0,005^{* *}$ & 0,558 & 0,534 \\
\hline Naturaleza de las prestaciones & $-0,700$ & 7,000 & $0,016^{*}$ & $-0,102$ & 0,096 \\
\hline Alcance & 0,203 & 2,400 & 0,137 & 0,102 & 0,028 \\
\hline Familiaridad con la tecnología & 0,477 & 4,601 & $0,044^{*}$ & 0,354 & 0,228 \\
\hline Canal de información digital & 0,545 & 2,669 & 0,094 & 0,294 & 0,217 \\
\hline Recursos de la web social & $-0,328$ & 1,048 & 0,369 & $-0,234$ & $-0,105$ \\
\hline
\end{tabular}

** -value $<0,01$ - * -value $<0,05$

Fuente. Elaboración propia. 
Con una capacidad explicativa del $74 \%$, el modelo propone interpretar la divulgación de información sobre la dimensión social de la RSC de forma tal de llegar a los diferentes públicos de interés con una mayor contribución de categorías si se dispone de un canal de información digital en las entidades del tipo mutual. Los resultados de una investigación dirigida a los clientes de una empresa estadounidense mostraron que el nivel de conocimiento de las actividades de responsabilidad social de la empresa se vincula positivamente con el uso de todos los canales de comunicación, por lo cual resulta de interés facilitar el desarrollo y la aplicación de estas tecnologías para la interacción con los grupos de interés (Lee, Zhang y Abitbol, 2019). La validación del modelo se efectuó a través de un análisis discriminante con un valor de Lambda de Wilks = 0,4 lo que indica un buen poder discriminante de las variables consideradas más contributivas por el análisis de regresión categórica.

La variable independiente identidad legal se asocia de forma positiva con la variable explicada - Sig.= 0,005 - con un coeficiente de 0,708 ; esto significa que en la medida en que la organización tenga una estructura societaria tipo mutualista será más proclive a la divulgación de contenidos sobre la dimensión social de RSC. La variable independiente naturaleza de las prestaciones se asocia de forma negativa con la variable explicada - Sig. $=0,016-$ considerando que, a medida que la naturaleza de las prestaciones tiende a ser financiadora, estas presentarán más contenidos sobre la dimensión social de RSC.

Dada la relación entre la RSE con los comportamientos de los clientes (Heesup, Jongsik, KyungSik y Hyungshin, 2020), resulta notoria tanto en la escasa presencia digital como en el número de entidades con contenidos específicos que aún no se valora la RSC en su vinculación con los comportamientos de los grupos de interés y, puesto que es necesario optimizar las demandas prestacionales de sus afiliados por el contexto inflacionario actual, esta resulta una estrategia viable para promover la sostenibilidad de la entidad.
A diferencia de las variables mencionadas, alcance no resulta significativa en el modelo $-p$-value > 0,05-; esta falta de influencia del tamaño de la entidad en la publicación de contenidos se observa diferente respecto a la investigación que encuentra a las organizaciones no lucrativas del sector sanitario estadounidense con mayor tamaño, pero con un menor nivel de divulgación de contenidos sobre RSC (Tremblay-Boire y Prakash, 2015). De acuerdo con el modelo obtenido, la primera hipótesis solo puede ser parcialmente asumida en la importancia que revisten los factores organizativos, pues dos de los tres factores tecnológicos propuestos son significativos, mientras que el alcance geográfico de la entidad no lo es.

La variable independiente familiaridad con la tecnología se asocia de forma positiva con la variable explicada -Sig. $=0,044-$. Esta asociación implica que las entidades que desarrollen su familiaridad con la tecnología de forma más intensiva generarán mejores contenidos sobre la dimensión social de RSC, pero a un ritmo más lento. A diferencia de la variable mencionada, canal informativo digital y recursos de la web social no resultan significativas $-p$-value $>0,05-$, por lo cual se asume parcialmente la segunda hipótesis de relevancia de los factores tecnológicos en la publicación digital de la dimensión social de RSC.

Entre los dos tipos de factores se observa que aquellos de mayor peso y en mayor número corresponden a los factores organizativos $y$, en menor medida, a los tecnológicos, de modo que proporcionan así una característica atribuible a su conformación y estructura más que a la dotación y los conocimientos sobre los avances tecnológicos. El mayor número de variables que responden a los factores organizativos respalda la idea de la importancia dada a la RSC según las características de la entidad en la que se desarrolle, más allá de los adelantos tecnológicos que se puedan incorporar. 
Bajo la guía de la literatura científica para la selección de factores relevantes y los resultados obtenidos del modelo, se asumen parcialmente las hipótesis planteadas inicialmente. Los factores relacionados con la identidad legal de la organización, así como la naturaleza de las prestaciones, es decir, aquellas variables referidas a la estructura organizativa, y la familiaridad con las tecnologías como factor tecnológico, explican el comportamiento de la comunicación sobre la dimensión social de RSC para las entidades vinculadas con los servicios de salud en, aproximadamente, las tres cuartas partes de los casos.

\section{Discusión y conclusiones}

$\mathrm{E}_{\mathrm{p}}^{\mathrm{n}}$ n este trabajo se analizaron los contenidos publicados en internet sobre la adquisición de hábitos saludables, conductas éticas y de compromiso con la sociedad por entidades no lucrativas vinculadas con servicios de salud de Mar del Plata a fin de determinar los factores —organizativos y tecnológicos- que influyen en la comunicación de la dimensión social de RSC. El primer resultado, preocupante, es la baja importancia que asigna el sector a la visibilidad de su organización a través de las tecnologías de internet. Con apenas un porcentaje mayor a la mitad de las organizaciones sin fines de lucro que prestan o financian servicios de salud en la localidad, la invisibilidad de sus acciones impacta también sobre voluntarios, donantes, proveedores, afiliados $\mathrm{y}$ otros grupos de interés que usan intensiva $\mathrm{y}$ extensivamente las tecnologías, además de excluir a la juventud dentro de su área de influencia.

El segundo resultado preocupante es el escaso número de entidades, entre las que disponen de presencia digital, con presentación de contenidos para la concientización de los grupos de interés sobre la RSC, como, por ejemplo, la promoción y prevención de la salud, proyectos sociales y de voluntariado, entre otras acciones. Aquellos que publican estos contenidos manifiestan su naturaleza intrínseca, mientras que para aquellos que no lo hacen, estas acciones, si ocurren, permanecen desconocidas tanto al interior como al exterior de la entidad, de manera que obstruyen una vía genuina para entablar un vínculo entre la entidad y las partes interesadas.
Sobre la determinación de los factores, los resultados se pueden dividir en dos partes. Una, referida a los que ejercen influencia en la presencia de dichos contenidos, y otra con aquellos factores que no contribuyen a explicar la divulgación del tema. Entre los que mejor explican la publicación sobre RSC se encuentran los factores identidad legal, naturaleza de las prestaciones y familiaridad con la tecnología.

En resumen, las entidades que publican contenidos sobre compromiso social, es decir, la dimensión social de la RSC, son mutuales, financiadoras de las prestaciones de sus asociados y con un vasto conocimiento de la tecnología para la provisión de una identidad a través de dominios específicos en el ámbito digital, ofreciendo así información sobre el cuidado de la salud a sus públicos de interés. Por otra parte, los factores basados en el alcance geográfico de la entidad, la disponibilidad de canales informativos y los recursos de la web social no resultan de influencia en la intención de las entidades de ofrecer información para contribuir a la adopción de hábitos y conductas saludables.

El estudio brinda un aporte empírico con respecto a la importancia de la naturaleza de las entidades antes que en su desarrollo tecnológico para la divulgación de información de relevancia sobre RSC, lo cual lleva el foco a las particularidades de cada tipo de organización antes que a las tecnologías de gestión y su implementación para la comunicación de su quehacer en materia de compromiso social. 
Con las problemáticas de estas entidades fundadas en la necesidad de optimizar las demandas prestacionales y a fin de ofrecer un servicio de calidad sostenible para todos sus afiliados en un contexto de alta inflación y elevada demanda de servicios médicos, cada vez más sofisticados y onerosos, la incorporación de la comunicación sobre las acciones de RSC en los medios digitales resultaría contributivo para el desarrollo y la consolidación del vínculo con sus grupos de interés. Esto constituiría un primer paso en la concientización sobre los hábitos y las conductas saludables, así como en fomentar el interés por participar en los proyectos sociales y programas de voluntariado que desarrolla la entidad.

Las líneas de trabajos futuros se inscriben en la visibilización a través de ránquines de la sostenibilidad de este tipo de entidades acerca de su comunicación digital a fin de promover no solo la divulgación de su compromiso con la comunidad y el ambiente, sino, además, la cooperación entre entidades y la mayor participación de las partes interesadas en dichas acciones.

\section{Referencias}

Adhepeau, J. L. M. (2013). Responsabilidad social corporativa (RSC) y teoría de los stakeholders en el sector del petróleo de Costa de Marfil. Prisma Social: Revista de Investigación Social, (10), 303-331.

Ajina, A. S.; Japutra, A.; Nguyen, B.; Alwi, S. F. S.; Al-Hajla, A. H. (2019). The importance of CSR initiatives in building customer support and loyalty. Asia Pacific Journal of Marketing and Logistics, 31(3), 691-713. DOI: https://doi.org/10.1108/ apjml-11-2017-0284

Alvarado-Herrera, A.; Schlesinger-Díaz, M. W. (2008). Dimensionalidad de la responsabilidad social empresarial percibida y sus efectos sobre la imagen y la reputación: una aproximación desde el modelo de Carroll. Estudios Gerenciales, 24(108), 37-59. DOI: https://doi.org/10.1016/S01235923(08)70043-3

Álvarez, I. G.; Sánchez, I. G.; Domínguez, L. R. (2009). La eficacia del gobierno corporativo y la divulgación de información en internet. Investigaciones Europeas de Dirección y Economía de la Empresa, 15(1), 109-135. DOI: https://doi.org/10.1016/ S1135-2523(12)60081-1
Anheier, H. K. (2013). The nonprofits of 2025. Stanford Social Innovation Review, 11(2), 18-19.

Baue, B.; Murninghan, M. (2011). The accountability web: weaving corporate accountability and interactive technology. Journal of Corporate Citizenship, (41), 27-49. DOI: https:// doi.org/10.9774/GLEAF.4700.2011.sp.00004

Belló, M.; Becerril-Montekio, V. M. (2011). Sistema de salud de Argentina. Salud Pública de México, 53, 96-109. DOI: https:// doi.org/10.1590/S0036-36342011000500003

Benavides-Delgado, J.; Fernández-Fernández, J. L.; de VivarFontelo, A. (2012). Glosario sobre responsabilidad social para la investigación y el debate terminológico. Madrid: Universidad Pontificia de Comillas de Madrid. Recuperado de https://bit. ly/2Soq3WP

Berger, A. A. (2020). Media and communication research methods: an introduction to qualitative and quantitative approaches (5a ed.). Los Ángeles: Sage Publication.

Bowen, H. R. (1953). Social responsibilities of the businessman. Iowa: University of Iowa Press.

Carroll, A. B. (1979). Un modelo conceptual tridimensional de desempeño corporativo. The Academy of Management Review, 4(4), 497-505. DOI: https://doi.org/10.5465/ amr.1979.4498296

Castaño-González, E. J. (2011). Comunicar la responsabilidad social, una opción de éxito empresarial poco explorada. Revista Lasallista de Investigación, 8(2), 173-186. Recuperado de https://bit.ly/2T6QEbf

Du, S.; Bhattacharya, C.; Sen, S. (2010), Maximizing business returns to corporate social responsibility (CSR): the role of CSR communication. International Journal of Management Reviews, 12(1), 8-19. DOI: https://doi.org/10.1111/j.14682370.2009.00276.x

Duverger, M. (1981). Los métodos de análisis de documentos y la técnica del análisis de contenido. Métodos de las ciencias sociales. Barcelona: Ariel.

Džupina, M.; Džupinová, Z. (2019). Dimensions of CSR in online communication of pharmaceutical companies: a comparative study. International Journal of Entrepreneurial Knowledge, 7(2), 41-52. DOI: https://doi.org/10.37335/ijek.v7i2.92

Freeman, E. (1984). Strategic management: a stakeholder approach. Boston: Pitman.

Gazzola, P.; Ratti, M.; Amelio, S. (2017). CSR and sustainability report for nonprofit organizations. An Italian best practice. Management Dynamics in the Knowledge Economy, 5(3), 355376. DOI: https://doi.org/10.25019/MDKE/5.3.03

Heesup, H.; Jongsik Y.; Kyung-Sik, L.; Hyungshin B. (2020). Impact of corporate social responsibilities on customer responses and brand choices. Journal of Travel \& Tourism Marketing, 37(3), 302-316. DOI: https://doi.org/10.1080/1 0548408.2020.1746731 
Horrach, P.; Socias-Salvà, A. (2011). La actitud de las empresas de economía solidaria frente a la divulgación de información sobre sostenibilidad desde el prisma de la teoría de los stakeholders o grupos de interés. Revista de Contabilidad, 14(1), 267-297. DOI: https://doi.org/10.1016/S11384891(11)70035-8

Izquierdo, R.J. S.; Grañana, I. V. (2005). La responsabilidad social corporativa (RSC) y su gestión integrada. Ciriec-España, Revista de Economía Pública, Social y Cooperativa, 53, 137-161.

Koinig I.; Diehl S.; Mueller B. (2017) Health communication and integrated corporate social responsibility. En S. Diehl; M. Karmasin; B. Mueller; R. Terlutter; F. Weder (Eds.) Handbook of integrated CSR communication. CSR. (471-494). Cham: Springer. DOI: https://doi.org/10.1007/978-3-319-447001_26

Lee, S. Y.; Zhang, W.; Abitbol, A. (2019). What makes CSR communication lead to CSR participation? Testing the mediating effects of CSR associations, CSR credibility, and organization-public relationships. Journal of Business Ethics, 157(2), 413-429. DOI: https://doi.org/10.1007/s10551017-3609-0

Mateos-Espejel, L.; Estrada-Rodríguez, J. L.; Hernández-Ruiz, H. M. (2020). Competitividad y responsabilidad social desde la comunicación de la ciencia. Revista Venezolana de Gerencia, 25(91), 995-1013. DOI: https://doi.org/10.37960/rvg. v25i91.33178

McMahon, D.; Seaman, S.; Lemley, D. A. (2015). The adoption of websites by nonprofits and the impact on society. Technology in Society, 42, 1-8. DOI: https://doi.org/10.1016/j. techsoc.2015.01.001

Moreno, A.; Capriotti, P. (2006).La comunicación de las empresas españolas en sus webs corporativas. Análisis de la información de responsabilidad social, ciudadanía corporativa y desarrollo sostenible. ZER: Revista de Estudios de Comunicación, 11(21), 47-62.

Moreno, A.; Capriotti, P. (2009). Comunicación sobre RSE, ciudadanía y sostenibilidad en la web, Revista de Gestión de la Comunicación, 13(2), 157-175. DOI: https://doi. org/10.1108/13632540910951768

Muñoz-Martín, J. (2013). Ética empresarial, responsabilidad social corporativa (RSC) y creación de valor compartido (CVC). Revista de Globalización, Competitividad y Gobernabilidad 7(3), 76-88.

Organización Mundial de la Salud. (1986). Ottawa Charter for Health Promotion. Recuperado de https://bit.ly/3vOG7ik

Pardinas, F. (2005). Metodología y técnicas de investigación en ciencias sociales. México: Siglo XXI.
Rodríguez-Cala, A.; Calle-Rodríguez, C.; Grunembaun, Z.; Pons-Rodríguez, A.; Benavente-Moreno, Y.; Durán-García, N. (2017). Responsabilidad social corporativa en los hospitales catalanes: ¿qué nos dicen sus webs? Revista de Comunicación y Salud, 7(1), 13-28. DOI: https://doi.org/10.35669/ revistadecomunicacionysalud.2017.7(1).13-28

Sánchez-Hervás, D.; Subiela-Hernández, B. (2016). La comunicación de la responsabilidad social corporativa en las organizaciones españolas con mejor reputación. Sphera Publica, 2(16), 39-53. Recuperado de https://bit.ly/3qo9LtG

Seguí-Mas, D.; Sarasa-Pérez, C.; Baviera-Puig, M. (2012). La comunicación de las políticas de RSC en las cooperativas de crédito: análisis a través del estudio del caso. Sociedad y Utopía, (40), 383-402.

Sigliano, A. (2013). Experiencia de una red de servicios de salud integrados: la Asociación Mutual de Protección Familiar en Argentina. Washington: Organización Panamericana de Salud. Recuperado de https://bit.ly/3d87XPV

Tapia-Frade, A.; Caerols-Mateo, R. (2015). Simetría y asimetría informativa en página web: el caso de las empresas del IBEX 35 y las ONG. Un análisis comparado. Trípodos, (36), 133150. Recuperado de https://bit.ly/3gRgoBG

Tremblay-Boire, J.; Prakash, A. (2015). Accountability.org: online disclosures by US nonprofits. Voluntas: International Journal of Voluntary and Nonprofit Organizations, 26(2), 693-719. DOI: https://doi.org/10.1007/s11266-014-9452-3

Vargas-Sánchez, A.; Vaca-Acosta, R. M. (2005). Responsabilidad socialcorporativaycooperativismo: vínculosypotencialidades. Revista de Economía Pública, Social y Cooperativa, (53), 241260. Recuperado de https://bit.ly/3wQyEAC

Waters, R. D.; Ott, H. K. (2014). Corporate social responsibility and the nonprofit sector: assessing the thoughts and practices across three nonprofit subsectors. Public Relations Journal, 8(3), 1-18.

Wirth, H.; Kulczycka, J.; Hausner, J.; Koński, M. (2016). Corporate social responsibility: communication about social and environmental disclosure by large and small copper mining companies. Resources Policy, 49, 53-60. DOI: https:// doi.org/10.1016/j.resourpol.2016.04.007 